OPEN ACCESS

Edited by:

Helieh S. Oz,

University of Kentucky, United States

Reviewed by:

Qiuxiang Zhang,

Jiangnan University, China

Yi Cai,

Sichuan Agricultural University, China

${ }^{*}$ Correspondence:

Gang Liu

gangle.liu@gmail.com

Jun Fang

fangjun1973@hunau.edu.cn

Specialty section This article was submitted to

Food Microbiology,

a section of the journal

Frontiers in Microbiology

Received: 29 October 2018

Accepted: 14 March 2019

Published: 03 April 2019

Citation:

Ma Y, Ding S, Liu G, Fang J, Yan W, Duraipandiyan V, Al-Dhabi NA

Esmail GA and Jiang H (2019) Egg

Protein Transferrin-Derived Peptides

IRW and IQW Regulate Citrobacter

rodentium-Induced

Inflammation-Related Microbial

and Metabolomic Profiles.

Front. Microbiol. 10:643.

doi: 10.3389/fmicb.2019.00643

\section{Egg Protein Transferrin-Derived Peptides IRW and IQW Regulate Citrobacter rodentium-Induced, Inflammation-Related Microbial and Metabolomic Profiles}

\author{
Yong Ma ${ }^{1}$, Sujuan Ding ${ }^{1}$, Gang Liu',2*, Jun Fang ${ }^{1 *}$, Wenxin Yan ${ }^{1}$, \\ Veeramuthu Duraipandiyan ${ }^{3}$, Naif Abdullah Al-Dhabi ${ }^{3}$, Galal Ali Esmail ${ }^{3}$ and \\ Hongmei Jiang ${ }^{1}$
}

${ }^{1}$ College of Bioscience and Biotechnology, Hunan Agricultural University, Changsha, China, ${ }^{2}$ Hunan Provincial Key Laboratory of Animal Nutritional Physiology and Metabolic Process, CAS Key Laboratory of Agro-ecological Processes in Subtropical Region, National Engineering Laboratory for Pollution Control and Waste Utilization in Livestock and Poultry Production, Institute of Subtropical Agriculture, Chinese Academy of Sciences, Changsha, China, ${ }^{3}$ Department of Botany and Microbiology, College of Sciences, King Saud University, Riyadh, Saudi Arabia

Bioactive peptides that target the gastrointestinal tract can strongly affect the health of animals and humans. This study aimed to evaluate the abilities of two peptides derived from egg albumin transferrin, IRW and IQW, to treat enteritis in a mouse model of Citrobacter rodentium-induced colitis by evaluating serum metabolomics and gut microbes. Forty-eight mice were randomly assigned to six groups: basal diet (CTRL), intragastric administration Citrobacter rodentium (CR), basal diet with 0.03\%IRW (IRW), CR with $0.03 \% \mathrm{IRW}(\mathrm{IRW}+\mathrm{CR})$, basal diet with $0.03 \% \mathrm{IQW}(\mathrm{IQW})$ and $\mathrm{CR}$ with $0.03 \%$ IQW (IQW+CR). CR administration began on day 10 and continued for 7 days. After 14 days of IRW and IQW treatment, serum was collected and subjected to a metabolomics analysis. The length and weight of each colon were measured, and the colon contents were collected for 16 srRNA sequencing. The colons were significantly longer in the CR group, compared to the CTRL group. A serum metabolomics analysis revealed no significant difference in microbial diversity between the six groups. Compared with the CTRL group, the proportions of Firmicutes and Actinobacteria species decreased significantly and the proportions of Bacteroidetes and Proteobacteria species increased in the CR group. There were no significant differences between the CTRL and other groups. The serum metabolomics analysis revealed that Infected by $C R$ increased the levels of oxalic acid, homogentisic acid and prostaglandin but decreased the levels of L-glutamine, L-acetyl carnitine, 1-methylhistidine and gentisic acid. Therefore, treatment with IRW and IQW was shown to regulate the intestinal microorganisms associated with colonic inflammation and serum metabolite levels, thus improving intestinal health.

Keywords: IRW, IQW, Citrobacter rodentium, inflammation, microbial, metabolomic 


\section{INTRODUCTION}

Citrobacter rodentium (CR) is a natural mouse pathogen that can be used to cause intestinal inflammation replaced enteropathogenic Escherichia coli (EHEC) and enteropathogenic E. coli (EPEC) (Hart et al., 2008). Although CR is a nonmotile pathogen, it can infect the host by passing through the intestinal mucosa to reach the intestinal epithelial cells. It adheres to the intestinal epithelial cells through surface structures called fimbriae when the CR started to infect the host (Mundy et al., 2003; Hart et al., 2008). These fimbriae can mediate infections in target cells that result in disorders of the host immune system. Therefore, the fimbriae are widely considered an important pathogenic factor in several diseases, particularly urinary, genital, and gastrointestinal infections (Petty et al., 2010). CR successfully colonizes intestinal epithelial cells, where it causes significant increases in the levels of cytokines and infiltrating immune cells in the intestinal environment (Collins et al., 2014). Consequently, infected intestinal epithelial cells promote the apical expression of antimicrobial peptides and inducible nitric oxide synthase, thereby modulating the inflammatory signal to produce NO, which inhibits CR (Vallance et al., 2002; Lopez et al., 2016). Although CR induces inflammatory infiltration, proinflammatory factor production, intestinal mucosal injury, and similar processes associated with inflammatory bowel disease in mice, this pathogen does not cause clinical diarrhea in humans (MacDonald et al., 2003). It may be an ideal model for mice but may not translate to human colonic inflammation, as it does not cause diarrhea in man (Zhang W. et al., 2015).

Many studies have provided substantial evidence indicating the regulatory roles of active peptides in intestinal inflammation (Kvidera et al., 2017; Eissa et al., 2018; Lv et al., 2018). The results of mouse experiments showed that porcine $\beta$-defensin 2 might improve mucosal lesions and extracellular permeability by targeting the NF- $\kappa \mathrm{B}$ pathway and could thus alleviate inflammatory enteritis (Han et al., 2015). Cathelicidin-related antimicrobial peptide (CRAMP) restore body weight loss caused by ulcerative colitis and maintain colonic epithelial integrity (Li et al., 2018). Cathelicidin-BF can effectively inhibit the phosphorylation of NF- $\mathrm{B}$ and increase the intestinal barrier function to reduce inflammation (Zhang H. et al., 2015). Egg protein transferrin-derived peptides, such as IRW and IQW, have been shown to provide effective relief of cardiovascular disease symptoms. The intact tripeptide structure of these molecules can alleviate endothelial inflammation and oxidative stress (Chen et al., 2017). IRW and IQW have also been shown to alleviate inflammatory responses induced by tumor necrosis factor (TNF) and may therefore be useful as nutraceuticals (Majumder et al., 2013). IRW can inhibit the TNF- $\alpha$-induced expression of both ICAM-1 and VCAM-1, whereas IQW can only inhibit the expression of ICAM-1. Treatment with IRW and IQW also inhibits the expression of antioxidant enzymes and promotes the activity of superoxide dismutase (SOD), catalase (CAT), and glutathione peroxidase (GPx) (Liu et al., 2018; Ma et al., 2018). Therefore, we hypothesized that IRW and IQW could reduce intestinal damage and thus slow the inflammatory process. This study mainly analyzed the levels of serum metabolites in a
$C R$-induced model of IBD in response to these two peptides, as well as the effects on microbial diversity and composition in the colonic contents.

\section{MATERIALS AND METHODS}

\section{Animal and Experimental Design}

The Animal Care and Use Committees of Hunan Agricultural University provided approval for the experiments. IRW and IQW (Ontores, Zhejiang, China) were dissolved in sterile water at $4^{\circ} \mathrm{C}$ until the solution temperature reached equilibrium with the ambient temperature. Both peptides were synthetic and had purity levels of $99 \%$. A CR bacterial solution was mixed with glycerol at a ratio of $3: 7$ and stored at $-80^{\circ} \mathrm{C}$ before inoculation into Luria Bertani broth to generate an activation culture before gavage. The mature CR culture was centrifuged at $4000 \mathrm{rpm}$ for $10 \mathrm{~min}$, and the supernatant was mixed with physiological saline to yield a $5 \times 10^{9} \mathrm{CFU} / \mathrm{ml}$ bacterial suspension that was set aside (Guan et al., 2016). Forty-eight mice (average body weight: $23 \mathrm{~g}$; age: 8 weeks) were randomly assigned to six groups ( $n=8$ per group): basal diet (CTRL), basal diet with intragastric administration of $0.1 \mathrm{~mL}$ bacterial suspension (CR), basal diet with $0.03 \%$ IRW (IRW), CR with $0.03 \%$ IRW (IRW+CR), basal diet with $0.03 \%$ IQW (IQW) and CR with $0.03 \%$ IQW (IQW+CR) (Liu et al., 2018). All mice in one group were housed and fed in seperate cages. The cages were stored in a sterile environment under a 12 -h light-dark cycle, relative humidity of $53 \%$, and temperature of $24^{\circ} \mathrm{C}$. After 3 days of acclimation, IRW and IQW were fed to mice. CR administration began on day 10 and continued for 7 days. All mice were slaughtered on day 17 . The experimental process adhered strictly to the animal experimental guidelines. Serum samples were collected for metabolomics analysis. The length and weight of the colon were measured, and a section was collected for a morphological analysis. The colon contents were also collected for 16 s rDNA sequencing.

\section{Histopathology}

Colon fragments were rinsed with saline and fixed in 12\% formalin. The fixed colon fragments were dehydrated using an ethanol gradient and embedded in paraffin. Ten-micrometer sections were stained with hematoxylin and eosin according to standard protocols. The samples were observed under blind conditions using an optic Olympus BX41 microscope (Münster, Germany).

\section{Serum Metabolomics}

The serum samples were thawed at room temperature and 100$\mu \mathrm{L}$ aliquots were transferred into centrifuge tubes $(1.5 \mathrm{~mL})$. All serum samples were extracted using $300 \mu \mathrm{L}$ of methanol and mixed with $10 \mu \mathrm{L}$ of an internal standard $(3.1 \mathrm{mg} / \mathrm{mL}, \mathrm{DL}-\mathrm{o}-$ chlorophenylalanine). The samples were then vortexed for $30 \mathrm{~s}$ and centrifuged at $12000 \mathrm{rpm}$ and $4^{\circ} \mathrm{C}$ for $15 \mathrm{~min}$. ACQUITYTM UPLC -QTOF analysis system and Waters ACQUITY UPLC HSS T3 chromatographic column $(2.1 \mathrm{~mm} \times 100 \mathrm{~mm}, 1.8$ covering $\mathrm{m})$ were used for LC-MS detection. The following chromatographic 
separation conditions were applied: column temperature, $40^{\circ} \mathrm{C}$; mobile phase $\mathrm{A}$, water $+0.1 \%$ formic acid; mobile phase $\mathrm{B}$ : acetonitrile $+0.1 \%$ formic acid; flow rate: $0.35 \mathrm{~mL} / \mathrm{min}$; injection volume: $6 \mu \mathrm{L}$. The data were first transformed to CDF files using CDF bridge and imported into XCMS software for peak picking, peak alignment, peak filtering and peak filling. The data, including the retention time (RT), MZ, observations (samples) and peak intensity, were normalized using Excel 2007.

\section{S rDNA and Illumina MiSeq Sequencing}

The colon contents were collected from all mice immediately after sacrifice. The V3-V4 region of the 16sRNA gene was sequenced in the 48 content samples. Microbial DNA was extracted from the colon contents using the QIAamp DNA Stool Mini Kit (Qiagen, Hilden, Germany). The microbial V3-V4 region was amplified by PCR using the primers 5'-ACTCCTACGGGAGGCAGCA and $3^{\prime}$-GGACT ACHVGGGTWTCTAAT. Amplicons were extracted from a $2 \%$ agarose gel using the TIANgel MIdi Purification Kit (TIANGEN BIOTECH, Beijing, China) and were subsequently purified. For detailed experimental steps on Illumina MiSeq sequencing, please see our previous article (Zhu et al., 2018); The general data analysis was performed by a commercial company (Novogene, Beijing, China).

\section{Correlation Analysis Between Differential Metabolite Levels and Genus-Level Intestinal Microbes}

SPSS 16.0 was used to conduct a correlative analysis of the proportions of microorganisms in each genus and details of the metabolic differences among all samples. GraphPad Prism was used to conduct a regression analysis of the results from the correlation analysis and draw graphs.

\section{Data Analysis}

All data are presented as means \pm standard errors of the means (SEM) and were analyzed using SPSS 16.0 software. Differences between mean values were evaluated using a one-way analysis of variance. If dissimilarities were detected, Tukey's multiple comparisons test was used. A $P$ value $<0.05$ was considered to indicate a significant difference.

\section{RESULTS}

\section{Effects of IRW and IQW on Changes in the Colon}

Infected by CR induced ulcers and other inflammatory reactions that caused mucosal damage in the colon and reduced the colon length. The average colon length in the CTRL group was $9.36 \pm 1.68 \mathrm{~cm}$, compared to $7.38 \pm 0.61 \mathrm{~cm}$ in the CR group. In other words, CR treatment significantly reduced the colon length $(P<0.05)$, whereas co-treatment with IRW and IQW maintained the colon length $(P>0.05)$ (Figure 1A). However, the colon weights did not differ between the six groups $(P>0.05)$ (Figure 1B). More severe histological damage was observed in colon tissues from the CR group, compared with the CTRL group, as demonstrated by broad disruption of the tissue architecture and the disappearance of intestinal crypts and goblet cells. Compared with the CTRL group, colon tissue from the CR group exhibited more extensive cellular infiltration (Figure 1C).

\section{Diversity of Bacterial Community of Colonic Contents}

Table 1 presents the original readings obtained by sequencing the V3-V4 region of 16s rRNA from each sample of colon contents. The sample size we sequenced is sufficient to reflect the abundance of microorganisms. The OTU (Operational Taxonomic Units) number of microbes in the colon is about 1200 (Figure 2). Raw reads and Clean Reads in the CR group were lower than other groups (Table 1). The six groups of goods coverage have reached more than $99 \%$. There were no statistically significant differences in community richness (chaol and ACE), community diversity (Shannon and Simpson) or species coverage (cargo coverage) (Table 1) $(P>0.05)$.

\section{Composition of Bacterial Communities in the Colon Contents}

We performed a taxonomic analysis of the sequencing results to determine the composition and community structure of different populations (or samples) at each classification level (e.g., domain, kingdom, gate, class, sequence, family, genus, species, OTU). At the phylum level, 10 phyla were detected at the highest abundance. Firmicutes, Bacteroidetes, Proteobacteria, Saccharibacteria and Actinobacteria were the most abundantly expressed phyla in all groups, accounting for 99\% of all sequencing results. In the CTRL group, Firmicutes (59.7\%), Bacteroidetes (27.5\%) and Proteobacteria (8.2\%) were the most abundant phyla (Figure 3), whereas Firmicutes (34.8\%), Bacteroidetes (41.4\%), Proteobacteria (17.2\%) and Saccharibacteria (4.2\%) were the most abundant phyla in the CR group (Figure 3). Firmicutes (56.1\%), Bacteroidetes (34.6\%), Proteobacteria (2.3\%) and Actinobacteria (5.6\%) were the most abundant phyla in the IRW group (Figure 3), while Firmicutes (47.8\%), Bacteroidetes (42.6\%), Proteobacteria (3.4\%) and Actinobacteria (4.7\%) were the most abundant phyla in the IRW+CR group (Figure 3). Firmicutes (77.1\%), Bacteroidetes (15.9\%) and Proteobacteria (5.0\%) were the most abundant phyla in the IQW group (Figure 3), while Firmicutes (58.4\%), Bacteroidetes (28.5\%) and Proteobacteria (5.0\%) were the most abundant in the IQW+CR group (Figure 3). Compared with the CTRL group, the CR group exhibited significantly lower percentages of Firmicutes $(59.7 \%$ vs. $34.8 \%)$ and Actinobacteria species $(2.3 \%$ vs. $1.7 \%)$ (Figure 3) but significantly higher percentages of Bacteroidetes $(27.5 \%$ vs. $41.4 \%)$, Proteobacteria $(8.2 \%$ vs. $17.2 \%$ ) and Saccharibacteria species (1.5\% vs. 4.2\%) (Figure 3). However, treatment with IRW and IQW restored the percentages of Firmicutes, Actinobacteria, Bacteroidetes, Proteobacteria and Saccharibacteria in CR animals to levels that did not 
A

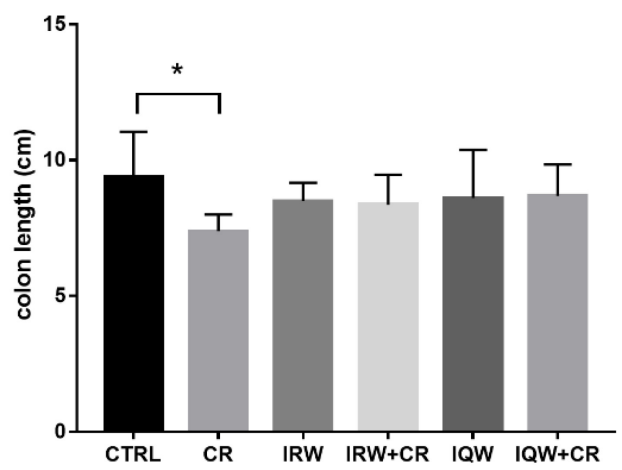

B

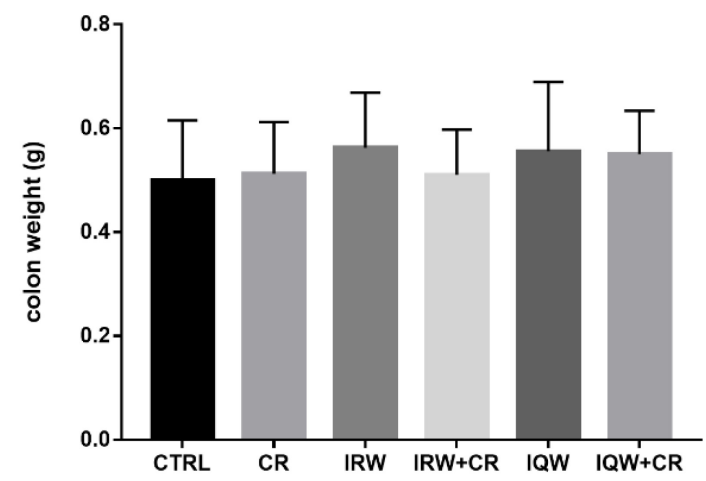

C

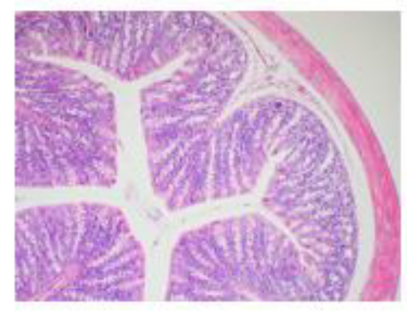

control

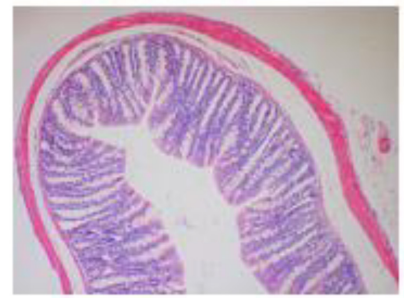

DSS

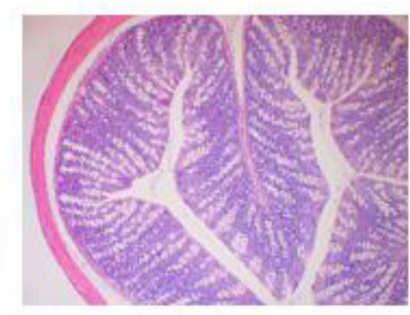

IRW

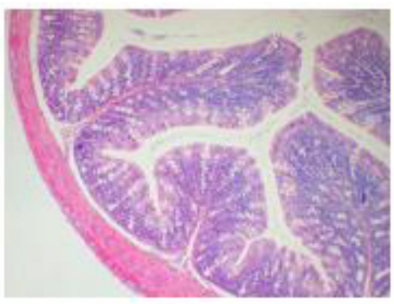

IRW+DSS

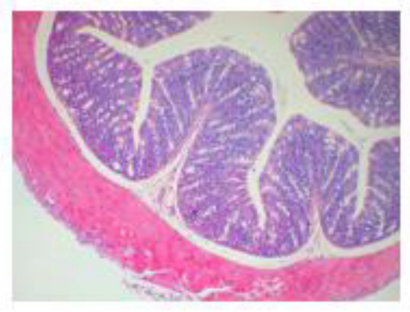

IQW

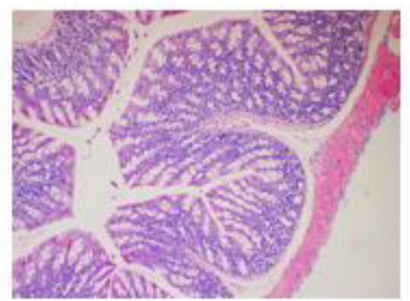

IQW+DSS

FIGURE 1 | Effects of IRW and IQW on (A) colon length and (B) colon weight. (C) Representative photomicrographs of colon sections stained with hematoxylin and eosin ( $\times 100$ magnification). Data are shown as means \pm standard errors of the means. $N=8,{ }^{*} P<0.05$.

TABLE 1 | Alpha diversity indices of fecal bacterial communities of mice.

\begin{tabular}{|c|c|c|c|c|c|c|}
\hline & CTRL & CR & IRW & IRW+CR & IQW & IQW+CR \\
\hline Raw_reads & $85186.75 \pm 2517.01$ & $82072.88 \pm 4917.86$ & $85372.38 \pm 1894.51$ & $88655.25 \pm 5995.91$ & $84431.88 \pm 5436.38$ & $89921.50 \pm 8418.42$ \\
\hline Clean_Reads & $80204.37 \pm 117.48$ & $78722.63 \pm 4523.63$ & $80060.75 \pm 268.08$ & $81782.25 \pm 5147.64$ & $80263.00 \pm 3899.80$ & $83648.50 \pm 7008.54$ \\
\hline Observed species & $1169.9 \pm 254.4167$ & $1372.5 \pm 261.9406$ & $1426.0 \pm 320.7420$ & $1413.9 \pm 199.1872$ & $1159.1 \pm 371.2440$ & $1437.4 \pm 212.9144$ \\
\hline Goods coverage & $0.9951 \pm 0.0016$ & $0.9935 \pm 0.0032$ & $0.9935 \pm 0.0009$ & $0.9938 \pm 0.0012$ & $0.9943 \pm 0.0014$ & $0.9929 \pm 0.0016$ \\
\hline Shannon & $6.6685 \pm 0.5191$ & $6.8589 \pm 0.4850$ & $6.5415 \pm 1.3152$ & $6.9744 \pm 0.5201$ & $5.8018 \pm 1.3015$ & $6.8660 \pm 0.52770$ \\
\hline Simpson & $0.9630 \pm 0.0132$ & $0.9608 \pm 0.0198$ & $0.9244 \pm 0.1000$ & $0.9685 \pm 0.0101$ & $0.9054 \pm 0.0756$ & $0.9699 \pm 0.0093$ \\
\hline Chao1 & $1324.5 \pm 302.4212$ & $1788.7 \pm 892.4182$ & $1612.2 \pm 329.4143$ & $1610.1 \pm 213.6898$ & $1343.5 \pm 387.572$ & $1697.8 \pm 230.7201$ \\
\hline ACE & $1357.3 \pm 302.5543$ & $1649.6 \pm 443.7628$ & $1668.9 \pm 325.3484$ & $1649.3 \pm 207.4422$ & $1395.4 \pm 391.6893$ & $1735.7 \pm 250.6430$ \\
\hline
\end{tabular}

differ significantly from the CTRL group (Figure 3). Figure 4 lists the 10 most abundant genera in the colonic contents. Notably, the percentage of Lactobacillus decreased and the percentages of Helicobacter and Odoribacter increased in the CR group, compared with the CTRL group (Figure 4). Similarly, IRW and IQW treatment restored the normal percentages of Lactobacillus, Helicobacter and Odoribacter species in CR animals. 


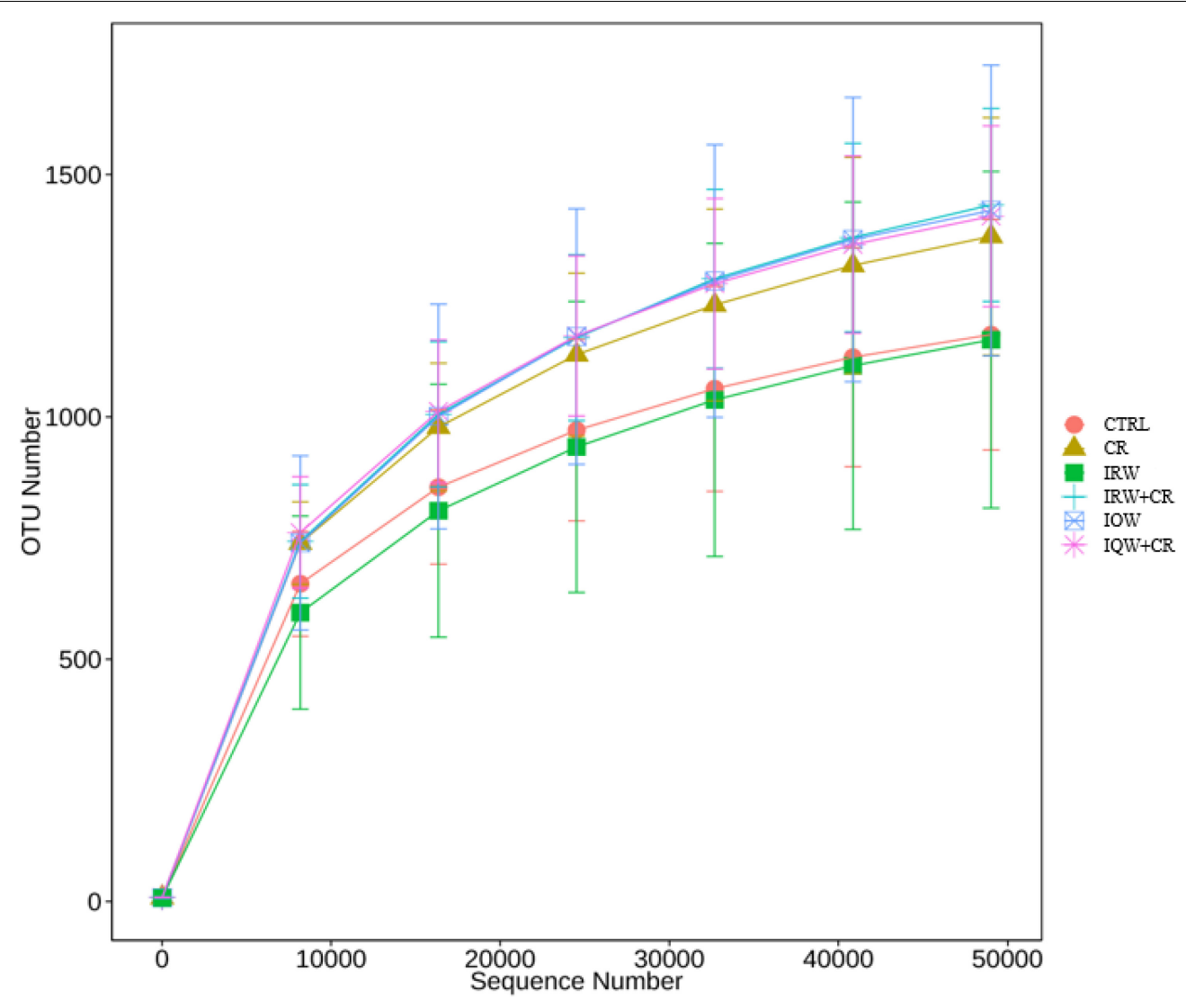

FIGURE 2 | Pan-species analysis curve corresponding to the total OTU number and sample size.

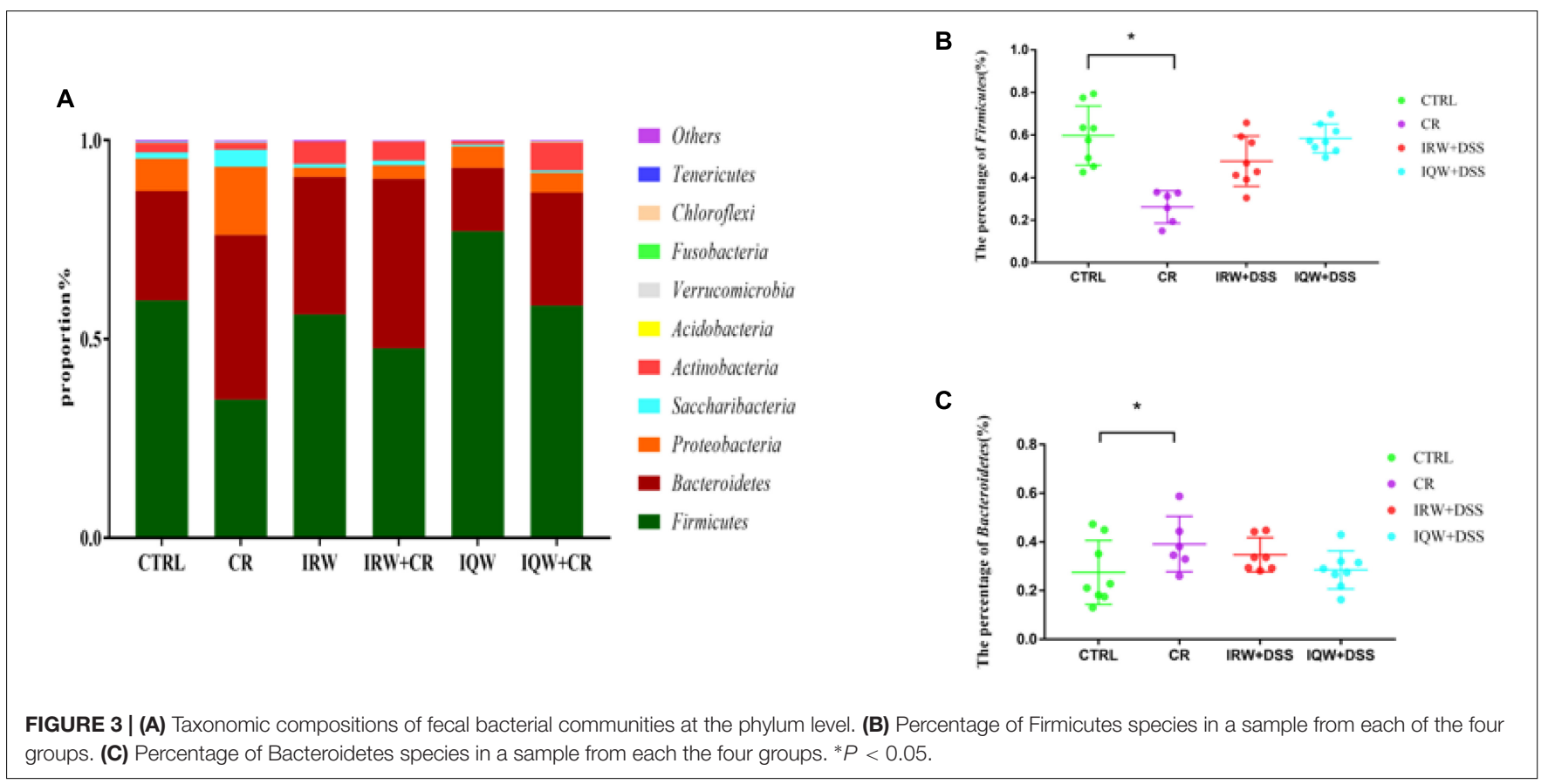



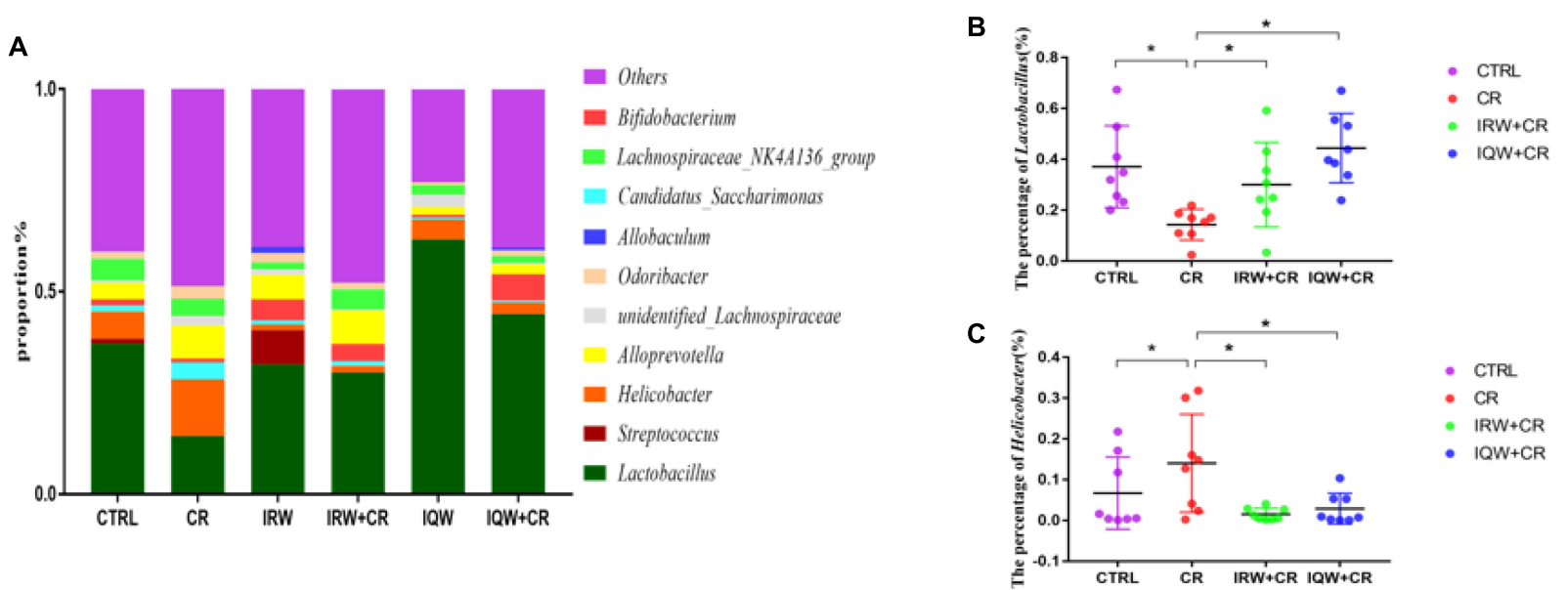

FIGURE 4 | (A) Taxonomic compositions of the fecal bacterial communities at the genus level. (B) Percentage of Lactobacillus species in a sample from each of the four groups. (C) Percentage of Helicobacter species in a sample from each of the four groups. ${ }^{*} P<0.05$.

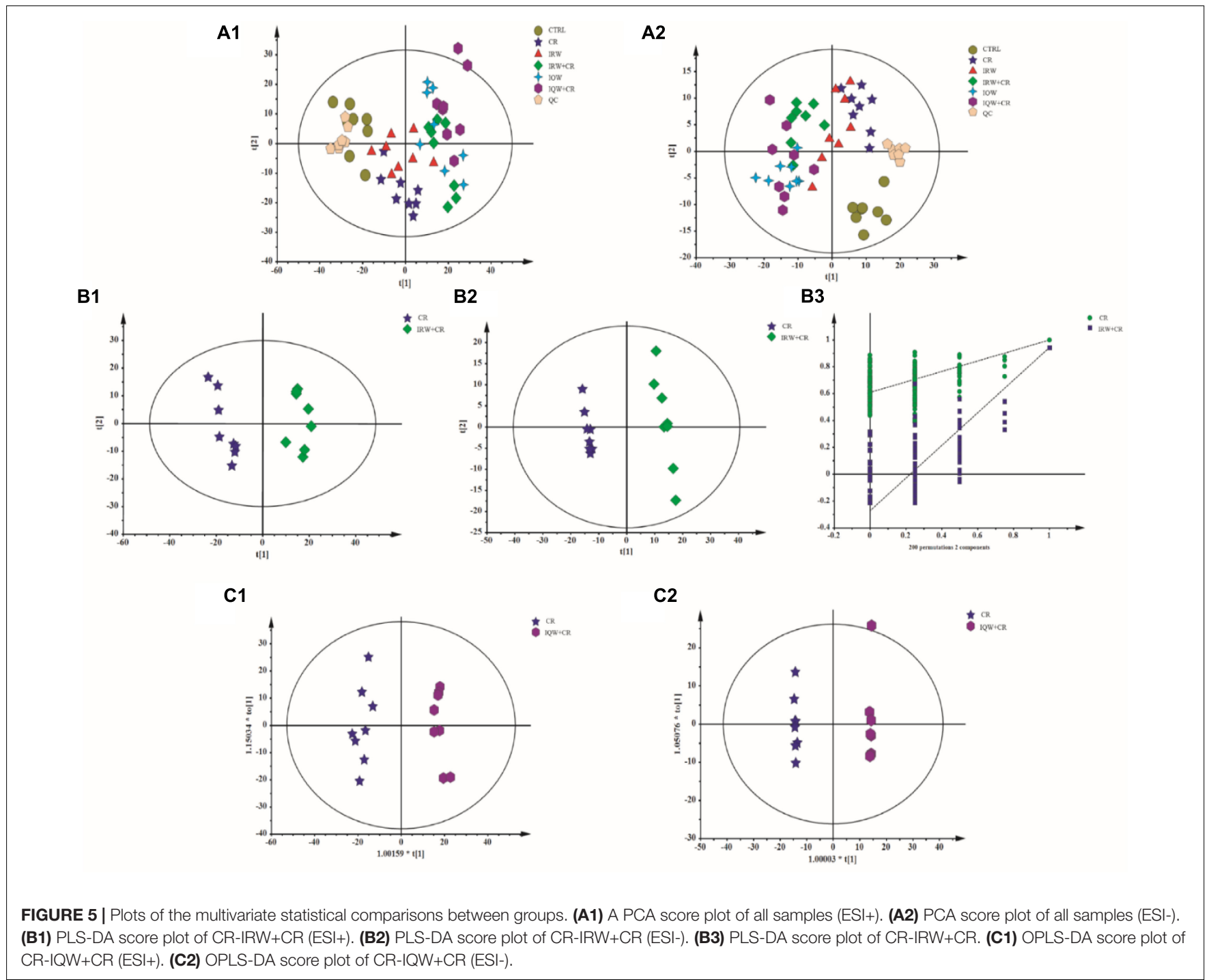




\section{Analysis of Serum Metabolomics}

To identify differences between the six groups, a PCA (Principal Component Analysis) modeling method was used to analyze the serum samples. In this analysis, six principal components were obtained in the positive mode, and the performance characteristics of this multivariate model in the positive ion mode were as follows: $R^{2} X=0.705, Q^{2}=0.591$. Nine principal components were obtained in the negative mode. Similarly, the performance characteristics of the model in the negative ion mode were as follows: $R^{2} X=0.531, Q^{2}=0.189$. In addition, the total PCA score demonstrates that the scatters of the six groups were completely separated in both the ESI+ (Figure 5A1) and ESI- modes (Figure 5A2). There were 10 metabolic differences between the CR and CTRL groups, including four in the ESI+ mode and six in the ESI- mode (Table 2). The results of a plasma metabolite analysis revealed decreased levels of oxalic acid, allantoin, homogentisic acid, $\alpha$-linolenic Acid, prostaglandin D3 and lysoPC and increased amounts of L-glutamine, gentisic acid, L-acetylcarnitine and 1-methylhistidine in the CR group. However, normal amounts of L-glutamine, gentisic acid, L-acetylcarnitine and 1-methylhistidine were observed in the IRW+CR and IQW+CR groups (Table 2).

When the CR group received IRW treatment, we further adopted a supervised multidimensional statistical method, namely the partial least square discriminant analysis (PLSDA), to conduct statistical analysis of the two groups of samples. The model quality parameters were two principal components in the positive mode, $R^{2} X=0.449, R^{2} Y=0.967$, $Q^{2}=0.904$, and two principal components in the negative mode, $\mathrm{R}^{2} \mathrm{X}=0.34, \mathrm{R}^{2} \mathrm{Y}=0.998, Q^{2}=0.942$. The PLS-DA loading plot demonstrates the complete separation of the scatters of the two groups (Figures $\mathbf{5 B 1}, \mathbf{B 2}$ ) due to differences in the detected metabolites. When the CR group received IQW treatment, the OPLS-DA method was again used for the modeling analysis. One principal component and one orthogonal component, $\mathrm{R}^{2} \mathrm{X}=0.481, \mathrm{R}^{2} \mathrm{Y}=0.979, Q^{2}=0.918$, were obtained in the positive mode. One principal component and two orthogonal components, $R^{2} X=0.472, R^{2} Y=1, Q^{2}=0.941$, were obtained in the negative mode. The OPLS-DA scoring diagrams are shown in Figures 5C1,C2. In order to verify whether the model is "overfitting", the model is sorted (Figure 5B3), and the results show that the model is reliable.

\section{Correlation Analysis Between Differential Metabolites and Genus-Level Intestinal Microbes}

The correlations between different metabolites in the serum metabolome results and the profiles of genus-level intestinal microbes. Gentisic acid, L-glutamine, 1-methylhistidine, lysoPC and L-acetylcarnitine were found to associate significantly with Lactobacillus. Specifically, negative correlations were detected between Lactobacillus and gentisic acid (Pearson correlation: $-0.347, P=0.016$ ) (Figure 6A), L-glutamine (Pearson correlation: $-0.412, P=0.004)$ (Figure 6B), 1methylhistidine (Pearson Correlation: $-0.438, P=0.002$ ) (Figure 6C), L-acetylcarnitine (Pearson correlation: -0.338 , $P=0.019$ ) (Figure 6E). However, a positive correlation was observed between Lactobacillus and LysoPC (Pearson correlation: $0.328, P=0.023$ ) (Figure 6D).

\section{DISCUSSION}

Citrobacter rodentium causes severe inflammatory infections in the mouse colon (Guan et al., 2016). These infections cause mucosal damage, reduce the colonic length, increased the colonic wall thickness, and cause hyperemia and even ulceration (Liu et al., 2015). Some studies also suggest that shortening of the colon is closely related to histological changes and even suggest that this morphological parameter indicates the degree of colonic inflammation (Yuge et al., 2014; Oz, 2017a). In our study, the colon lengths of mice infected intragastrically with CR were significantly shorter than those of normal mice, and this change was eliminated in the presence of IRW and IQW. The shortening of colon length may be an early warning of

TABLE 2 | Metabolomic changes in the plasma in the CTRL, CR, IRW+CR and IQW+CR.

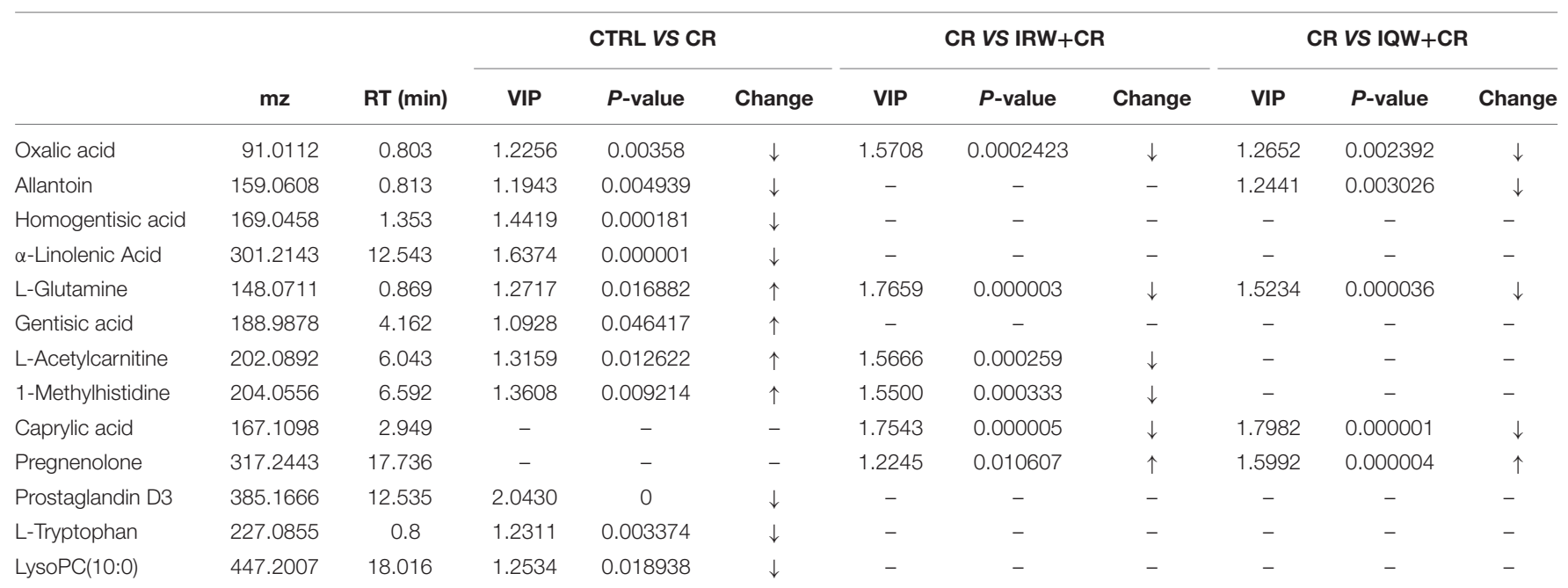


A

Gentisic acid vs. Lactobacillusata

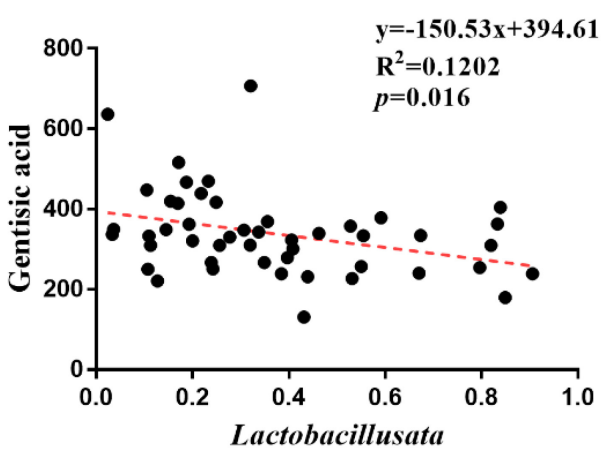

C

1-Methylhistidine vs. Lactobacillus

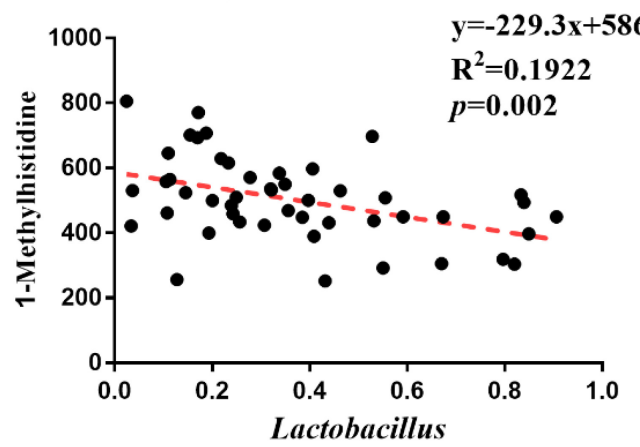

B

L-Glutamine vs. Lactobacillus

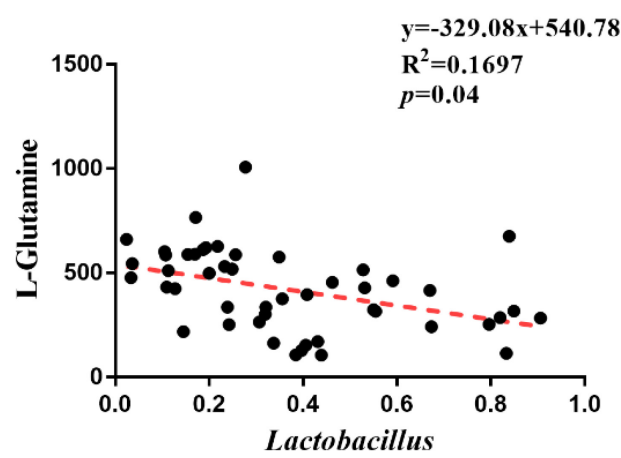

D

LysoPC vs. Lactobacillus

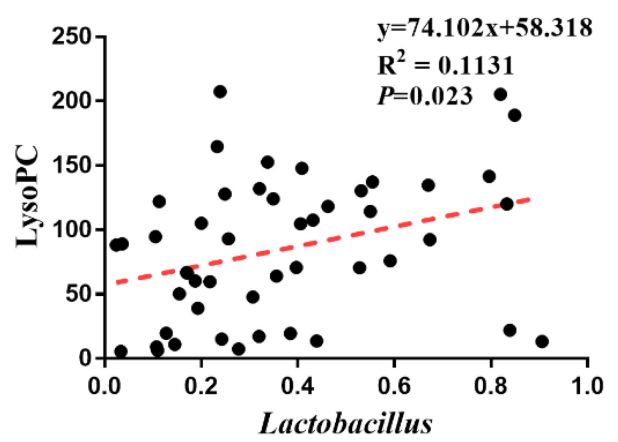

E

L-Acetylcarnitine vs. Lactobacillus

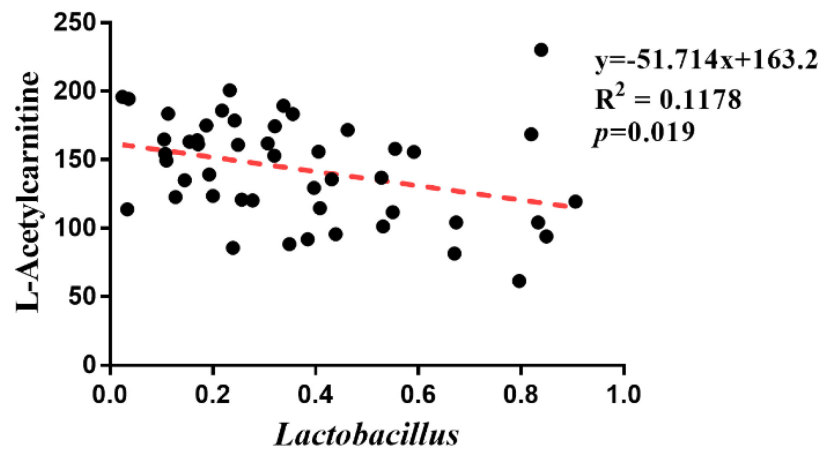

FIGURE 6 | Analysis of correlations between differential metabolites and genus-level intestinal microbes. (A) Correlation analysis between Lactobacillus and gentisic acid. (B) Correlation analysis between Lactobacillus and L-glutamine. (C) Correlation analysis between Lactobacillus and 1-methylhistidine. (D) Correlation analysis between Lactobacillus and LysoPC. (E) Correlation analysis between Lactobacillus and L-acetylcarnitine.

inflammation. A large number of experimental results indicate that colonic inflammation is accompanied by a decrease in colon length and other different degrees of morphological damage (Gearry et al., 2009; Gadaleta et al., 2011). Accordingly, IRW and IQW treatment appears to play a role in colonic inflammation (Jiao et al., 2019).

Mouse fecal microbes may be contaminated, so we try to be aseptic as possible during the sampling process and the samples are stored in sterile, enzyme-free EP tubes. Our microbiological sequencing results are consistent with the composition of the gut microbiota in most inflammatory studies, so the possibility of contamination is low. The intestinal microbes change significantly at the time of intestinal inflammation. However, different degrees of inflammation lead to different results in dissimilar individuals; for example, IBD ecological disorders differ widely (Walker et al., 2011; Weichselbaum and 
Klein, 2018). Notably, colonic inflammation commonly results in decreases in microbial diversity and the proportion of Firmicutes, as well as an increase in the proportion of Proteobacteria (Matsuoka and Kanai, 2015; Hong and Piao, 2018). In this study, the lack of significant changes in microbial diversity may be attributable to large differences in diversity within the group, a consequence of poor homogeneity among the mice. Fortunately, the proportion of Firmicutes was lower and the proportion of Proteobacteria was higher in the CR group, compared to the CTRL group. CR played a role in establishing a model of colonic inflammation. The proportions of Firmicutes and Proteobacteria returned to normal, however, in mice treated with IRW and IQW, which demonstrates the usefulness of these treatments. Lactobacillus and Bifidobacterium are the most commonly used probiotics (Pace et al., 2015; Azad et al., 2018b). Some studies have shown that Lactobacillus degrades pro-inflammatory factors secreted in milk proteins, thus eliminating inflammation (von Schillde et al., 2012; Yin et al., 2018). Furthermore, a study by Hormannsperger et al. (2013) found that Lactobacillus not only degraded pro-inflammatory chemokines and reduced immune cell infiltration in colonic inflammation (Di Cerbo and Palmieri, 2013; Lightfoot et al., 2015) but could also be used to identify protective microbial structures and even in new drug applications (Hormannsperger et al., 2013; Li and Gui, 2018; Zhang and Gui, 2018). This study also confirmed that the proportions of Lactobacillus and Bifidobacterium were significantly reduced in the CR group but returned to normal after treatment with IRW and IQW. Helicobacter spp. cause more severe intestinal inflammation and injury via M1 macrophages (Krakowiak et al., 2015; Azad et al., 2018a; Ding et al., 2019). Other studies have shown that Helicobacter can improve ROS levels by inducing regeneration synthesis and neutrophil infiltration of the epithelium both in vivo and in vitro (Davies et al., 1994; Bagchi et al., 1996; Mannick et al., 1996). The proportion of inflammatory Helicobacter species was significantly increased in the CR group, whereas this increase was inhibited by IRW and IQW. Accordingly, these peptides improved the intestinal condition.

When serum metabolomics were measured in normal and colitis mice, significant differences were observed (Schicho et al., 2010). Some studies have found that inflammatory infections can alter the structure of the gut microbiota and regulate the corresponding serum amino acids accordingly. As the proportion of Helicobacter increased, the concentration of tryptophan decreased, consistent with the changing trends in our study results. This finding suggests that Helicobacter can inhibit tryptophan metabolism (Gadaleta et al., 2011). Catabolic enzymes and indoleamine 2,3-dioxygenase (IDO) can metabolize tryptophan to kynurenine (Munn et al., 1998; Puccetti and Grohmann, 2007), and tryptophan metabolism was shown to affect immune modulation in recent studies (Romani et al., 2008; Fu et al., 2018). The tryptophan concentration may be inversely proportional to the disease activity and CRP level; in other words, a decrease in tryptophan is associated with inflammation (Hisamatsu et al., 2012). Hashimoto et al. found that caprylic acid could inhibit transcription of the gene encoding IL-8 in Caco-2 cells (Hoshimoto et al., 2002), thereby inhibiting the chemotaxis and proliferation of inflammatory cells. This phenomenon was also found to increase mitochondrial respiration in inflammatory cells (Hecker et al., 2014; Oz, 2017b). The expression of caprylic acid improved significantly after IRW and IQW were administered to CR mice, indicating that these protein transferrin-derived peptides play a role in CR-induced intestinal inflammation. Specifically, these peptides alter the composition of gut microbes that regulate metabolites. In turn, these metabolites increase the level of caprylic acid and thus improve the symptoms of inflammation. Lactobacillus can have good antibacterial activity against Clostridium (Monteiro et al., 2019), and can well reduce the oxidative stress caused by high-fat diet (Hsu et al., 2019). Lactobacillus has been shown to produce SCFA (short chain fatty acid) from L-glutamine (Botta et al., 2017). In humans, SCFA can regulate immune and metabolic functions in multiple tissues and organs (Ohira et al., 2017; Bin et al., 2018). Therefore, we believe that the amount of L-glutamine decreases (due to increased consumption) as the number of Lactobacillus increases, leading to a negative correlation between these parameters. The correlation between these species and metabolites indicates that the strain can promote the production of some inhibitory substances or can use these substances to produce metabolites to inhibit inflammation, thereby alleviating the inflammation of the colon.

\section{CONCLUSION}

In conclusion, supplementation of IRW and IQW improves the morphological damage of the colon caused by CR, while regulating the microbial composition in the colon and altering the serum metabolites through changes in microbial composition. Finally, colonic inflammation is improved by changes in these metabolite components. Further studies are needed to clarify and confirm the benefits of IRW and IQW treatment in the host gastrointestinal tract and other organ systems.

\section{AUTHOR CONTRIBUTIONS}

YM and SD carried out the study and performed the statistical analysis. VD, NA-D, and GE provided assistance for the experiments. GL, JF, and HJ designed the research. YM, SD, and WY prepared the first draft of the manuscript. GL and JF read and revised the manuscript.

\section{FUNDING}

This research was supported by National Natural Science Foundation of China (Nos. 31672457 and 31772642), National Key Research and Development Program of China (2016YFD0500504 and 2016YFD0501201), Ministry of Agricultural of the People's Republic of China (2015-Z64 and 2016-X47), Local Science and Technology Development Project Guided by The Central Government (YDZX20184300002303 
and 2018CT5002), and Hunan Provincial Science and Technology Department (2017NK2322, 2018TP1031, 2016NK2101, 2016WK2008, 2016TP2005, and 2018WK4025), China Postdoctoral Science Foundation (2018M632963), and Double first-class construction project of Hunan Agricultural University (SYL201802003, YB2018007).

\section{REFERENCES}

Azad, M. A. K., Bin, P., Liu, G., Fang, J., Li, T., and Yin, Y. (2018a). Effects of different methionine levels on offspring piglets during late gestation and lactation. Food Funct. 9, 5843-5854. doi: 10.1039/c8fo01343h

Azad, M. A. K., Sarker, M., Li, T., and Yin, J. (2018b). Probiotic species in the modulation of gut microbiota: an overview. Biomed. Res. Int. 2018:9478630. doi: 10.1155/2018/9478630

Bagchi, D., Bhattacharya, G., and Stohs, S. J. (1996). Production of reactive oxygen species by gastric cells in association with Helicobacter pylori. Free Radic. Res. 24, 439-450. doi: 10.3109/10715769609088043

Bin, P., Azad, M. A. K., Liu, G., Zhu, D., Kim, S. W., and Yin, Y. (2018). Effects of different levels of methionine on sow health and plasma metabolomics during late gestation. Food Funct. 9, 4979-4988. doi: 10.1039/c8fo01477a

Botta, C., Acquadro, A., Greppi, A., Barchi, L., Bertolino, M., Cocolin, L., et al. (2017). Genomic assessment in Lactobacillus plantarum links the butyrogenic pathway with glutamine metabolism. Sci. Rep. 7:15975. doi: 10.1038/s41598017-16186-8

Chen, S., Jiang, H., Peng, H., Wu, X., and Fang, J. (2017). The utility of ovotransferrin and ovotransferrin-derived peptides as possible candidates in the clinical treatment of cardiovascular diseases. Oxid. Med. Cell Longev. 2017:6504518. doi: 10.1155/2017/6504518

Collins, J. W., Keeney, K. M., Crepin, V. F., Rathinam, V. A., Fitzgerald, K. A., Finlay, B. B., et al. (2014). Citrobacter rodentium: infection, inflammation and the microbiota. Nat. Rev. Microbiol. 12, 612-623. doi: 10.1038/nrmicro3315

Davies, G. R., Banatvala, N., Collins, C. E., Sheaff, M. T., Abdi, Y., Clements, L., et al. (1994). Relationship between infective load of Helicobacter pylori and reactive oxygen metabolite production in antral mucosa. Scand. J. Gastroenterol. 29, 419-424. doi: 10.3109/00365529409096832

Di Cerbo, A., and Palmieri, B. (2013). Lactobacillus paracasei subsp. Paracasei F19; a farmacogenomic and clinical update. Nutr. Hosp. 28, 1842-1850. doi: 10.3305/nutr

Ding, S., Fang, J., Liu, G., Veeramuthu, D., Naif Abdullah, A. D., and Yin, Y. (2019). The impact of different levels of cysteine on the plasma metabolomics and intestinal microflora of sows from late pregnancy to lactation. Food Funct. 10, 691-702. doi: 10.1039/c8fo01838c

Eissa, N., Hussein, H., Hendy, G. N., Bernstein, C. N., and Ghia, J. E. (2018). Chromogranin-A and its derived peptides and their pharmacological effects during intestinal inflammation. Biochem. Pharmacol. 152, 315-326. doi: 10.1016/j.bcp.2018.04.009

Fu, C., Guan, G., and Wang, H. (2018). The anticancer effect of sanguinarine: a review. Curr. Pharm. Des. 24, 2760-2764. doi: 10.2174/ 1381612824666180829100601

Gadaleta, R. M., van Erpecum, K. J., Oldenburg, B., Willemsen, E. C., Renooij, W., Murzilli, S., et al. (2011). Farnesoid X receptor activation inhibits inflammation and preserves the intestinal barrier in inflammatory bowel disease. Gut 60 , 463-472. doi: 10.1136/gut.2010.212159

Gearry, R. B., Irving, P. M., Barrett, J. S., Nathan, D. M., Shepherd, S. J., and Gibson, P. R. (2009). Reduction of dietary poorly absorbed short-chain carbohydrates (FODMAPs) improves abdominal symptoms in patients with inflammatory bowel disease-a pilot study. J. Crohns. Colitis 3, 8-14. doi: 10.1016/j.crohns. 2008.09.004

Guan, G., Wang, H., Chen, S., Liu, G., Xiong, X., Tan, B., et al. (2016). Dietary chitosan supplementation increases microbial diversity and attenuates the severity of Citrobacter rodentium infection in mice. Med. Inflamm. 2016:9236196.

Hart, E., Yang, J., Tauschek, M., Kelly, M., Wakefield, M. J., Frankel, G., et al. (2008). RegA, an AraC-like protein, is a global transcriptional regulator that

\section{ACKNOWLEDGMENTS}

The authors would like to extend their sincere appreciation to the Deanship of Scientific Research at King Saud University for its funding of this research through the Research Group Project No. RGP-213.

controls virulence gene expression in Citrobacter rodentium. Infect. Immun. 76, 5247-5256. doi: 10.1128/IAI.00770-08

Han, F., Zhang, H., Xia, X., Xiong, H., Song, D., Zong, X., et al. (2015). Porcine beta-defensin 2 attenuates inflammation and mucosal lesions in dextran sodium sulfate-induced colitis. J. Immunol. 194, 1882-1893. doi: 10.4049/jimmunol. 1402300

Hecker, M., Sommer, N., Voigtmann, H., Pak, O., Mohr, A., Wolf, M., et al. (2014). Impact of short- and medium-chain fatty acids on mitochondrial function in severe inflammation. JPEN J. Parent. Enteral Nutr. 38, 587-594. doi: 10.1177/ 0148607113489833

Hisamatsu, T., Okamoto, S., Hashimoto, M., Muramatsu, T., Andou, A., Uo, M., et al. (2012). Novel, objective, multivariate biomarkers composed of plasma amino acid profiles for the diagnosis and assessment of inflammatory bowel disease. PLoS One 7:e31131. doi: 10.1371/journal.pone.0031131

Hong, Z., and Piao, M. (2018). Effect of quercetin monoglycosides on oxidative stress and gut microbiota diversity in mice with dextran sodium sulphateinduced colitis. Biomed. Res. Int. 2018:8343052. doi: 10.1155/2018/8343052

Hormannsperger, G., von Schillde, M. A., and Haller, D. (2013). Lactocepin as a protective microbial structure in the context of IBD. Gut Microbes 4, 152-157. doi: $10.4161 /$ gmic. 23444

Hoshimoto, A., Suzuki, Y., Katsuno, T., Nakajima, H., and Saito, Y. (2002). Caprylic acid and medium-chain triglycerides inhibit IL- 8 gene transcription in Caco-2 cells: comparison with the potent histone deacetylase inhibitor trichostatin A. Br. J. Pharmacol. 136, 280-286. doi: 10.1038/sj.bjp.0704719

Hsu, C. L., Hou, Y. H., Wang, C. S., Lin, S. W., Jhou, B. Y., Chen, C. C., et al. (2019). Antiobesity and uric acid-lowering effect of Lactobacillus plantarum GKM3 in high-fat-diet-induced obese rats. J. Am. College Nutr. doi: 10.1080/07315724. 2019.1571454 [Epub ahead of print].

Jiao, H., Zhang, Q., Lin, Y., Gao, Y., and Zhang, P. (2019). The ovotransferrinderived peptide IRW attenuates lipopolysaccharide-induced inflammatory responses. Biomed. Res. Int. 2019:8676410. doi: 10.1155/2019/8676410

Krakowiak, M. S., Noto, J. M., Piazuelo, M. B., Hardbower, D. M., Romero-Gallo, J., Delgado, A., et al. (2015). Matrix metalloproteinase 7 restrains Helicobacter pylori-induced gastric inflammation and premalignant lesions in the stomach by altering macrophage polarization. Oncogene 34, 1865-1871. doi: 10.1038/ onc. 2014.135

Kvidera, S. K., Horst, E. A., Sanz Fernandez, M. V., Abuajamieh, M., Ganesan, S., Gorden, P. J., et al. (2017). Characterizing effects of feed restriction and glucagon-like peptide 2 administration on biomarkers of inflammation and intestinal morphology. J. Dairy Sci. 100, 9402-9417. doi: 10.3168/jds.201713229

Li, X. Y., and Gui, J. F. (2018). Diverse and variable sex determination mechanisms in vertebrates. Sci China Life Sci 61, 1503-1514. doi: 10.1007/s11427-0189415-7

Li, Y., Chu, X., Liu, C., Huang, W., Yao, Y., Xia, Y., et al. (2018). Exogenous murine antimicrobial peptide CRAMP significantly exacerbates ovalbumin-induced airway inflammation but ameliorates oxazolone-induced intestinal colitis in BALB/c mice. Hum. Vacc. Immunother. 14, 146-158. doi: 10.1080/21645515. 2017.1386823

Lightfoot, Y. L., Selle, K., Yang, T., Goh, Y. J., Sahay, B., Zadeh, M., et al. (2015). SIGNR3-dependent immune regulation by Lactobacillus acidophilus surface layer protein A in colitis. EMBO J. 34, 881-895. doi: $10.15252 /$ embj.201490296

Liu, B., Lin, Q., Yang, T., Zeng, L., Shi, L., Chen, Y., et al. (2015). Oat beta-glucan ameliorates dextran sulfate sodium (DSS)-induced ulcerative colitis in mice. Food Funct. 6, 3454-3463. doi: 10.1039/c5fo00563a

Liu, G., Yan, W., Ding, S., Jiang, H., Ma, Y., Wang, H., et al. (2018). Effects of IRW and IQW on oxidative stress and gut microbiota in dextran sodium sulfateinduced colitis. Cell Physiol. Biochem. 51, 441-451. doi: 10.1159/000495240 
Lopez, C. A., Miller, B. M., Rivera-Chavez, F., Velazquez, E. M., Byndloss, M. X., Chavez-Arroyo, A., et al. (2016). Virulence factors enhance Citrobacter rodentium expansion through aerobic respiration. Science 353, 1249-1253. doi: $10.1126 /$ science.aag3042

Lv, D., Xiong, X., Yang, H., Wang, M., He, Y., Liu, Y., et al. (2018). Effect of dietary soy oil, glucose, and glutamine on growth performance, amino acid profile, blood profile, immunity, and antioxidant capacity in weaned piglets. Sci. China Life Sci. 61, 1233-1242. doi: 10.1007/s11427-018-9301-y

Ma, Y., Yan, W., Ding, S., Fei, Y., Liu, G., and Fang, J. (2018). Effects of bioactive peptide on inflammatory bowel disease, focus on signal transduction and intestinal microbiota. Curr. Pharm. Des. 24, 2782-2788. doi: 10.2174/ 1381612824666180829103945

MacDonald, T. T., Frankel, G., Dougan, G., Goncalves, N. S., and Simmons, C. (2003). Host defences to Citrobacter rodentium. Int. J. Med. Microbiol. 293, 87-93. doi: 10.1078/1438-4221-00247

Majumder, K., Chakrabarti, S., Davidge, S. T., and Wu, J. (2013). Structure and activity study of egg protein ovotransferrin derived peptides (IRW and IQW) on endothelial inflammatory response and oxidative stress. J. Agric. Food Chem. 61, 2120-2129. doi: 10.1021/jf3046076

Mannick, E. E., Bravo, L. E., Zarama, G., Realpe, J. L., Zhang, X. J., Ruiz, B., et al. (1996). Inducible nitric oxide synthase, nitrotyrosine, and apoptosis in Helicobacter pylori gastritis: effect of antibiotics and antioxidants. Cancer Res. 56, 3238-3243.

Matsuoka, K., and Kanai, T. (2015). The gut microbiota and inflammatory bowel disease. Semin. Immunopathol. 37, 47-55. doi: 10.1007/s00281-014-0454-4

Monteiro, C., do Carmo, M. S., Melo, B. O., Alves, M. S., Dos Santos, C. I., Monteiro, S. G., et al. (2019). In vitro antimicrobial activity and probiotic potential of bifidobacterium and Lactobacillus against species of Clostridium. Nutrients 11:448. doi: 10.3390/nu11020448

Mundy, R., Pickard, D., Wilson, R. K., Simmons, C. P., Dougan, G., and Frankel, G. (2003). Identification of a novel type IV pilus gene cluster required for gastrointestinal colonization of Citrobacter rodentium. Mol. Microbiol. 48, 795-809. doi: 10.1046/j.1365-2958.2003.03470.x

Munn, D. H., Zhou, M., Attwood, J. T., Bondarev, I., Conway, S. J., Marshall, B., et al. (1998). Prevention of allogeneic fetal rejection by tryptophan catabolism. Science 281, 1191-1193. doi: 10.1126/science.281.5380.1191

Ohira, H., Tsutsui, W., and Fujioka, Y. (2017). Are short chain fatty acids in gut microbiota defensive players for inflammation and atherosclerosis? J. Atheroscler. Thromb. 24, 660-672. doi: 10.5551/jat.RV17006

Oz, H. S. (2017a). Chronic inflammatory diseases and green tea polyphenols. Nutrients 9:E561. doi: 10.3390/nu9060561

Oz, H. S. (2017b). Nutrients, infectious and inflammatory diseases. Nutrients 9:1085. doi: 10.3390/nu9101085

Pace, F., Pace, M., and Quartarone, G. (2015). Probiotics in digestive diseases: focus on Lactobacillus GG. Min. Gastroenterol. Dietol. 61, 273-292.

Petty, N. K., Bulgin, R., Crepin, V. F., Cerdeno-Tarraga, A. M., Schroeder, G. N., Quail, M. A., et al. (2010). The Citrobacter rodentium genome sequence reveals convergent evolution with human pathogenic Escherichia coli. J. Bacteriol. 192, 525-538. doi: 10.1128/JB.01144-09

Puccetti, P., and Grohmann, U. (2007). IDO and regulatory T cells: a role for reverse signalling and non-canonical NF-kappaB activation. Nat. Rev. Immunol. 7, 817-823. doi: 10.1038/nri2163

Romani, L., Fallarino, F., De Luca, A., Montagnoli, C., D’Angelo, C., Zelante, T., et al. (2008). Defective tryptophan catabolism underlies inflammation in mouse chronic granulomatous disease. Nature 451, 211-215. doi: 10.1038/ nature 06471

Schicho, R., Nazyrova, A., Shaykhutdinov, R., Duggan, G., Vogel, H. J., and Storr, M. (2010). Quantitative metabolomic profiling of serum and urine in DSS-induced ulcerative colitis of mice by (1)H NMR spectroscopy. J. Proteome Res. 9, 6265-6273. doi: 10.1021/pr100547y

Vallance, B. A., Deng, W., De Grado, M., Chan, C., Jacobson, K., and Finlay, B. B. (2002). Modulation of inducible nitric oxide synthase expression by the attaching and effacing bacterial pathogen Citrobacter rodentium in infected mice. Infect. Immun. 70, 6424-6435. doi: 10.1128/IAI.70.11.6424-6435.2002

von Schillde, M. A., Hormannsperger, G., Weiher, M., Alpert, C. A., Hahne, H., Bauerl, C., et al. (2012). Lactocepin secreted by Lactobacillus exerts antiinflammatory effects by selectively degrading proinflammatory chemokines. Cell Host Microbe 11, 387-396. doi: 10.1016/j.chom.2012.02.006

Walker, A. W., Sanderson, J. D., Churcher, C., Parkes, G. C., Hudspith, B. N., Rayment, N., et al. (2011). High-throughput clone library analysis of the mucosa-associated microbiota reveals dysbiosis and differences between inflamed and non-inflamed regions of the intestine in inflammatory bowel disease. BMC Microbiol. 11:7. doi: 10.1186/1471-2180-11-7

Weichselbaum, L., and Klein, O. D. (2018). The intestinal epithelial response to damage. Sci. China Life Sci. 61, 1205-1211. doi: 10.1007/s11427-018-9331-y

Yin, J., Li, Y., Han, H., Chen, S., Gao, J., Liu, G., et al. (2018). Melatonin reprogramming of gut microbiota improves lipid dysmetabolism in high-fat diet-fed mice. J. Pineal. Res. 65:e12524. doi: 10.1111/jpi.12524

Yuge, K., Takahashi, T., Khai, N. C., Goto, K., Fujiwara, T., Fujiwara, H., et al. (2014). Intramuscular injection of adenoviral hepatocyte growth factor at a distal site ameliorates dextran sodium sulfate-induced colitis in mice. Int. J. Mol. Med. 33, 1064-1074. doi: 10.3892/ijmm.2014.1686

Zhang, Q. Y., and Gui, J. F. (2018). Diversity, evolutionary contribution and ecological roles of aquatic viruses. Sci. China Life Sci. 61, 1486-1502. doi: 10.1007/s11427-018-9414-7

Zhang, H., Xia, X., Han, F., Jiang, Q., Rong, Y., Song, D., et al. (2015). CathelicidinBF, a novel antimicrobial peptide from Bungarus fasciatus, attenuates disease in a dextran sulfate sodium model of colitis. Mol. Pharm. 12, 1648-1661. doi: 10.1021/acs.molpharmaceut.5b00069

Zhang, W., Du, J. Y., Yu, Q., and Jin, J. O. (2015). Interleukin-7 produced by intestinal epithelial cells in response to Citrobacter rodentium infection plays a major role in innate immunity against this pathogen. Infect. Immun. 83, 3213-3223. doi: 10.1128/IAI.00320-15

Zhu, D., Ma, Y., Ding, S., Jiang, H., and Fang, J. (2018). Effects of melatonin on intestinal microbiota and oxidative stress in colitis mice. Biomed. Res. Int. 2018:2607679. doi: 10.1155/2018/2607679

Conflict of Interest Statement: The authors declare that the research was conducted in the absence of any commercial or financial relationships that could be construed as a potential conflict of interest.

Copyright (c) 2019 Ma, Ding, Liu, Fang, Yan, Duraipandiyan, Al-Dhabi, Esmail and Jiang. This is an open-access article distributed under the terms of the Creative Commons Attribution License (CC BY). The use, distribution or reproduction in other forums is permitted, provided the original author(s) and the copyright owner(s) are credited and that the original publication in this journal is cited, in accordance with accepted academic practice. No use, distribution or reproduction is permitted which does not comply with these terms. 